\title{
BVR $_{c} I_{c}$ Observations and Analysis of the Near-Contact Solar Type Eclipsing Binary, V530 Andromedae
}

\author{
Ronald G. Samec, ${ }^{1,2}$ Daniel Flaaten, ${ }^{1}$ James Kring, ${ }^{1,2}$ and Danny R. Faulkner ${ }^{2,3,4}$ \\ ${ }^{1}$ Astronomy Group, Department of Physics and Engineering, Bob Jones University, Greenville, SC 29614, USA \\ ${ }^{2}$ Lowell Observatory, Flagstaff, AZ 86001, USA \\ ${ }^{3}$ University of South Carolina, Lancaster, SC 29720, USA \\ ${ }^{4}$ Johnson Observatory, Petersburg, KY 41080, USA \\ Correspondence should be addressed to Ronald G. Samec; rsamec@bju.edu
}

Received 3 August 2013; Accepted 8 September 2013

Academic Editors: F. Arenou, A. Pamyatnykh, and A. Sesana

Copyright (C) 2013 Ronald G. Samec et al. This is an open access article distributed under the Creative Commons Attribution License, which permits unrestricted use, distribution, and reproduction in any medium, provided the original work is properly cited.

We present the first precision $U_{B V R} I_{c}$ light curves, an initial period study, and a simultaneous light curve solution for the nearcontact solar type eclipsing binary V530 And. Our observations were taken with the $0.81 \mathrm{~m}$ Lowell reflector on 27 and 29 September, 2011, with time being granted from the National Undergraduate Research Observatory (NURO). Our Wilson Devinney Program solution yields a semidetached, V1010 Oph configuration: the more massive component is filling its Roche lobe. The system is apparently approaching contact for the first time. It is not a classic Algol.

\section{History}

V530 And [2MASS J01274106 + 3351552, NSVS 6447718, TYC 2300-116-1, $\alpha(2000)=01$ h $27 \mathrm{~m} 41.050 \mathrm{~s}, \delta(2000)=$ $+33^{\circ} 51^{\prime} 55.47^{\prime \prime}$ ICRS, $\left.J=11.679, H=11.485, K=11.421\right]$ was discovered by Khruslov (2008) [1]. It was designated as EB with a 12.6-13.3 R-magnitude range (Min II $=13.0$ ) with the ephemeris:

$$
\text { J.D. Hel Min } \mathrm{I}=2451479.632 \mathrm{~d}+0.57723 \cdot E \text {. }
$$

Their light curve is given as Figure 1.

V530 And was observed by the Robotic Optical Transient Search Experiment [2] and found to have a period $=0.57721 \mathrm{~d}$ and a mean unfiltered magnitude of 12.769. A light curve amplitude of $0.633 \mathrm{mag}$ was determined. It was stated that the light curve was calculated to be in the Fourier region where $\beta$ Lyrae (EB) types are expected. Finally, V530 And appeared in the 80th namelist of variable stars (IBVS \#5969, 2011). It was also designated as an EB type. EB binaries are very close together producing distorted curves from tidal and Coriolis forces, but the stars are not in contact, so their light curves still maintain dissimilar eclipse depths.

\section{Observations}

This system was observed as a part of our student/professional collaborative studies of interacting binaries from data taken from NURO observations (National Undergraduate Research Observatory). Our light curves were taken with the Lowell $0.81 \mathrm{~m}$ reflector in Flagstaff with a CRYOTIGER cooled $\left(<-100^{\circ} \mathrm{C}\right)$ NASACAM and a $2048 \times 2048$ CCD chip with standard UBVR ${ }_{c} I_{c}$ filters. Observations were taken on 27 and 29 September, 2011, by Samec, Kring, and Faulkner. The time was awarded by the Lowell TAC as a part of the time allocated from NURO. Analyses were done by Flaaten and Samec. The individual observations included 92 in the $U$-filter, 93 in $B, 92$ in $V$, and 91 in $R$ and $I$. The standard error of a single observation was $3 \mathrm{mmag}$ in $U$ and $B, 2 \mathrm{mmag}$ in $V$ and $R$, and $4 \mathrm{mmag}$ in $I$. Our observations are given in Table 1, in delta magnitudes, $\Delta B, \Delta V, \Delta R_{c}$, and $\Delta I_{c}$ in the sense of variable minus comparison star. 


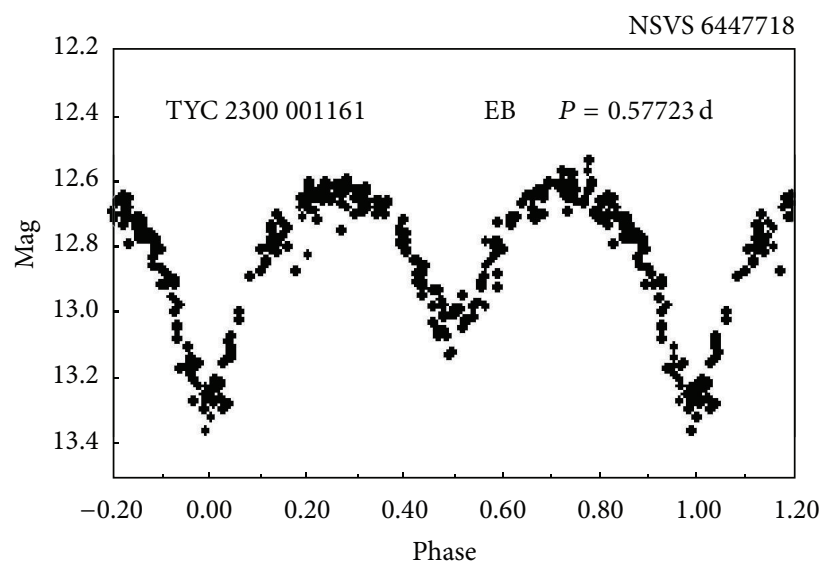

FIgURE 1: Light curve for V530 And from Khruslov (2008) [1].

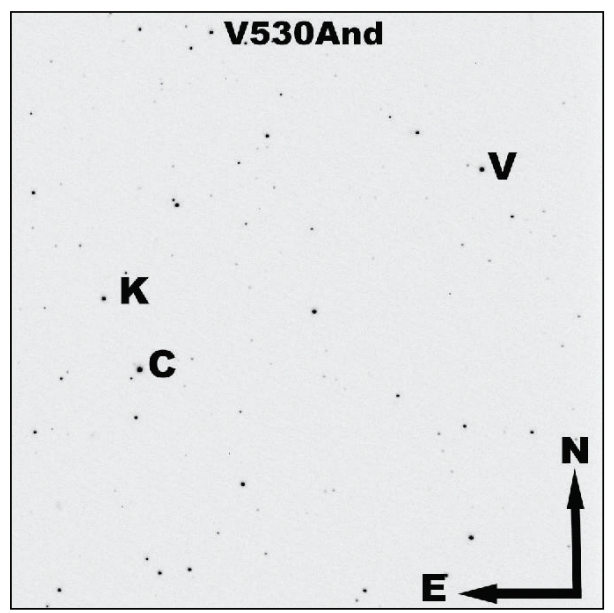

Figure 2: Finding chart, V530 And variable $(V)$, comparison $(C)$, and check $(K)$.

\section{Finding Chart}

Our comparison star $(C)$ was GSC 2300 0035, $[\alpha(2000)=$ $01 \mathrm{~h} 27 \mathrm{~m} 41.0660 \mathrm{~s}, \delta(2000)=+33^{\circ} 51^{\prime} 55.298^{\prime \prime} \mathrm{V}=$ $12.832, B-V=0.53$ Guide 9$]$. The check star $(K)$ was GSC $23000053,[\alpha(2000)=14$ h $05 \mathrm{~m} 13.8544 \mathrm{~s}, \delta(2000)=$ $+38^{\circ} 57^{\prime} 24.156^{\prime \prime} V=12.421, B-V=0.64$, Guide 9]. We include a finding chart of these stars and the variable $(V)$ in Figure 2.

\section{Period Determination}

Two mean times of minimum light were determined from our observations. These include HJD Min I = $2455832.74595( \pm 0.00044) \mathrm{d}$ and HJD Min II = $2455830.72806( \pm 0.00045) \mathrm{d}$. We determined 9 more times of low light from the NSVS catalog data set for object number 6447718. We combined this data with Khruslov's ephemeris to calculate the following new ephemeris (see Table 2 for the calculation):

J.D. Hel Min I = 2455832.7470 $( \pm 0.0170) \mathrm{d}$

$$
+0.577217( \pm 0.000003) \cdot E \text {. }
$$

The following is from our Wilson-Devinney program light curve solution:

$$
\begin{aligned}
& \text { J.D. Hel Min I= } 2455832.7468( \pm 0.0001) \mathrm{d} \\
& +0.576805( \pm 0.000001) \cdot E .
\end{aligned}
$$

Each time of minimum light and low light used in the calculation and depicted in Figure 3 is given in Table 2 along with their residuals. Equation (3) is from the programs iterative fit to the light curve data while (2) is a least squares fit to the minima. The NSVS minima are times of low light and carry large uncertainties. We include both ephemerides since they are the best we have at this time.

What we have here is an initial period study. The Wilson programs determination (3) may be better than the one determined from the minima. Further observations, over the course of 10 years or more, are needed to better determine a precision ephemeris and the nature of the period behavior of the system. 
TABLE 1: Photometry of V530 And.

\begin{tabular}{|c|c|c|c|c|c|c|c|c|c|}
\hline$\Delta U$ & $\begin{array}{c}U_{\mathrm{HJD}} \\
2455800+\end{array}$ & $\Delta U$ & $\begin{array}{c}U_{\mathrm{HJD}} \\
2455800+\end{array}$ & $\Delta U$ & $\begin{array}{c}U_{\mathrm{HJD}} \\
2455800+\end{array}$ & $\Delta U$ & $\begin{array}{c}U_{\mathrm{HJD}} \\
2455800+\end{array}$ & $\Delta U$ & $\begin{array}{c}U_{\mathrm{HJD}} \\
2455800+\end{array}$ \\
\hline 1.066 & 30.6497 & 1.269 & 30.7671 & 1.034 & 30.8610 & 1.384 & 32.6971 & 1.148 & 32.8347 \\
\hline 1.100 & 30.6569 & 1.224 & 30.7736 & 1.035 & 30.8669 & 1.425 & 32.7031 & 1.118 & 32.8411 \\
\hline 1.127 & 30.6642 & 1.143 & 30.7868 & 1.032 & 30.8728 & 1.546 & 32.7142 & 1.096 & 32.8470 \\
\hline 1.158 & 30.6714 & 1.133 & 30.7937 & 1.039 & 30.8794 & 1.640 & 32.7225 & 1.081 & 32.8529 \\
\hline 1.205 & 30.6796 & 1.114 & 30.8003 & 1.046 & 30.8853 & 1.727 & 32.7292 & 1.064 & 32.8587 \\
\hline 1.247 & 30.6874 & 1.093 & 30.8070 & 1.056 & 30.8912 & 1.758 & 32.7350 & 1.050 & 32.8645 \\
\hline 1.314 & 30.6958 & 1.287 & 32.6839 & 1.082 & 30.9016 & 1.775 & 32.7417 & 1.035 & 32.8728 \\
\hline 1.358 & 30.7035 & 1.324 & 32.6899 & 1.091 & 30.9075 & 1.776 & 32.7475 & 1.025 & 32.8786 \\
\hline 1.395 & 30.7108 & 1.384 & 32.6971 & 1.103 & 30.9133 & 1.781 & 32.7546 & 1.015 & 32.8857 \\
\hline 1.402 & 30.7174 & 1.425 & 32.7031 & 1.124 & 30.9193 & 1.756 & 32.7604 & 1.021 & 32.8915 \\
\hline 1.398 & 30.7244 & 1.546 & 32.7142 & 1.140 & 30.9251 & 1.704 & 32.7669 & 1.010 & 32.8974 \\
\hline 1.397 & 30.7309 & 1.640 & 32.7225 & 1.160 & 30.9310 & 1.619 & 32.7727 & 1.011 & 32.9032 \\
\hline 1.388 & 30.7383 & 1.133 & 30.7937 & 1.188 & 30.9381 & 1.549 & 32.7791 & 1.030 & 32.9093 \\
\hline 1.395 & 30.7449 & 1.114 & 30.8003 & 1.765 & 31.0202 & 1.473 & 32.7849 & 1.023 & 32.9151 \\
\hline 1.352 & 30.7536 & 1.093 & 30.8070 & 1.112 & 32.6514 & 1.401 & 32.7924 & 1.036 & 32.9210 \\
\hline 1.309 & 30.7602 & 1.083 & 30.8135 & 1.140 & 32.6587 & 1.340 & 32.7981 & 1.058 & 32.9268 \\
\hline 1.269 & 30.7671 & 1.079 & 30.8217 & 1.165 & 32.6646 & 1.290 & 32.8043 & 1.052 & 32.9335 \\
\hline 1.224 & 30.7736 & 1.057 & 30.8324 & 1.202 & 32.6713 & 1.257 & 32.8101 & 1.054 & 32.9393 \\
\hline 1.143 & 30.7868 & 1.048 & 30.8390 & 1.229 & 32.6773 & 1.220 & 32.8160 & 1.064 & 32.9454 \\
\hline 1.352 & 30.7536 & 1.042 & 30.8474 & 1.287 & 32.6839 & 1.194 & 32.8218 & 1.086 & 32.9512 \\
\hline \multirow[t]{2}{*}{1.309} & 30.7602 & 1.032 & 30.8532 & 1.324 & 32.6899 & 1.168 & 32.8283 & 1.123 & 32.9670 \\
\hline & & & & & & & & 1.134 & 32.9728 \\
\hline$\Delta B$ & $\begin{array}{c}B_{\mathrm{HJD}} \\
2455800+\end{array}$ & $\Delta B$ & $\begin{array}{c}B_{\mathrm{HJD}} \\
2455800+\end{array}$ & $\Delta B$ & $\begin{array}{c}B_{\mathrm{HJD}} \\
2455800+\end{array}$ & $\Delta B$ & $\begin{array}{c}B_{\mathrm{HJD}} \\
2455800+\end{array}$ & $\Delta B$ & $\begin{array}{c}B_{\mathrm{HJD}} \\
2455800+\end{array}$ \\
\hline 1.074 & 30.6520 & 1.131 & 30.7892 & 1.104 & 30.9215 & 1.741 & 32.7497 & 1.025 & 32.8667 \\
\hline 1.109 & 30.6593 & 1.108 & 30.7961 & 1.124 & 30.9274 & 1.729 & 32.7568 & 1.012 & 32.8750 \\
\hline 1.130 & 30.6666 & 1.087 & 30.8027 & 1.142 & 30.9333 & 1.718 & 32.7626 & 1.005 & 32.8808 \\
\hline 1.161 & 30.6738 & 1.073 & 30.8093 & 1.170 & 30.9404 & 1.641 & 32.7691 & 0.996 & 32.8879 \\
\hline 1.209 & 30.6820 & 1.058 & 30.8159 & 1.075 & 32.6452 & 1.567 & 32.7749 & 0.990 & 32.8937 \\
\hline 1.249 & 30.6897 & 1.048 & 30.8240 & 1.111 & 32.6536 & 1.490 & 32.7813 & 1.002 & 32.8996 \\
\hline 1.304 & 30.6982 & 1.028 & 30.8348 & 1.137 & 32.6609 & 1.422 & 32.7871 & 1.000 & 32.9054 \\
\hline 1.358 & 30.7059 & 1.020 & 30.8414 & 1.157 & 32.6669 & 1.351 & 32.7945 & 1.009 & 32.9115 \\
\hline 1.370 & 30.7132 & 1.016 & 30.8496 & 1.179 & 32.6735 & 1.297 & 32.8003 & 1.003 & 32.9173 \\
\hline 1.380 & 30.7198 & 1.010 & 30.8555 & 1.220 & 32.6795 & 1.251 & 32.8065 & 1.019 & 32.9232 \\
\hline 1.373 & 30.7267 & 1.009 & 30.8633 & 1.271 & 32.6862 & 1.220 & 32.8123 & 1.033 & 32.9290 \\
\hline 1.381 & 30.7333 & 1.011 & 30.8691 & 1.309 & 32.6921 & 1.190 & 32.8182 & 1.037 & 32.9357 \\
\hline 1.370 & 30.7407 & 1.012 & 30.8750 & 1.378 & 32.6994 & 1.158 & 32.8240 & 1.037 & 32.9415 \\
\hline 1.361 & 30.7472 & 1.012 & 30.8817 & 1.431 & 32.7054 & 1.143 & 32.8305 & 1.046 & 32.9476 \\
\hline 1.325 & 30.7560 & 1.027 & 30.8875 & 1.538 & 32.7164 & 1.122 & 32.8369 & 1.065 & 32.9534 \\
\hline 1.277 & 30.7626 & 1.033 & 30.8934 & 1.637 & 32.7247 & 1.077 & 32.8433 & 1.117 & 32.9692 \\
\hline 1.235 & 30.7694 & 1.059 & 30.9039 & 1.709 & 32.7314 & 1.063 & 32.8492 & 1.137 & 32.9750 \\
\hline 1.190 & 30.7760 & 1.068 & 30.9097 & 1.743 & 32.7372 & 1.055 & 32.8551 & & \\
\hline 1.160 & 30.7826 & 1.052 & 30.9156 & 1.746 & 32.7439 & 1.032 & 32.8609 & & \\
\hline$\Delta V$ & $\begin{array}{c}V_{\mathrm{HJD}} \\
2455800+ \\
\end{array}$ & $\Delta V$ & $\begin{array}{c}V_{\mathrm{HJD}} \\
2455800+ \\
\end{array}$ & $\Delta V$ & $\begin{array}{c}V_{\text {HJD }} \\
2455800+ \\
\end{array}$ & $\Delta V$ & $\begin{array}{c}V_{\mathrm{HJD}} \\
2455800+ \\
\end{array}$ & $\Delta V$ & $\begin{array}{c}V_{\text {HJD }} \\
2455800+ \\
\end{array}$ \\
\hline 1.128 & 30.6529 & 1.175 & 30.7901 & 1.121 & 30.9163 & 1.739 & 32.7504 & 1.072 & 32.8674 \\
\hline 1.157 & 30.6602 & 1.147 & 30.7970 & 1.142 & 30.9222 & 1.734 & 32.7575 & 1.057 & 32.8757 \\
\hline 1.181 & 30.6675 & 1.132 & 30.8036 & 1.168 & 30.9281 & 1.722 & 32.7633 & 1.045 & 32.8815 \\
\hline 1.218 & 30.6747 & 1.118 & 30.8103 & 1.180 & 30.9339 & 1.654 & 32.7697 & 1.042 & 32.8885 \\
\hline 1.270 & 30.6829 & 1.104 & 30.8168 & 1.124 & 32.6461 & 1.581 & 32.7755 & 1.040 & 32.8943 \\
\hline 1.320 & 30.6907 & 1.093 & 30.8250 & 1.144 & 32.6544 & 1.505 & 32.7820 & 1.041 & 32.9003 \\
\hline 1.378 & 30.6991 & 1.086 & 30.8277 & 1.173 & 32.6617 & 1.452 & 32.7878 & 1.045 & 32.9061 \\
\hline
\end{tabular}


TABle 1: Continued.

\begin{tabular}{|c|c|c|c|c|c|c|c|c|c|}
\hline 1.423 & 30.7068 & 1.073 & 30.8357 & 1.195 & 32.6677 & 1.376 & 32.7952 & 1.054 & 32.9122 \\
\hline 1.437 & 30.7141 & 1.061 & 30.8423 & 1.219 & 32.6744 & 1.328 & 32.8010 & 1.055 & 32.9180 \\
\hline 1.439 & 30.7207 & 1.056 & 30.8503 & 1.257 & 32.6803 & 1.281 & 32.8071 & 1.067 & 32.9239 \\
\hline 1.441 & 30.7277 & 1.053 & 30.8562 & 1.303 & 32.6870 & 1.243 & 32.8129 & 1.074 & 32.9297 \\
\hline 1.434 & 30.7342 & 1.046 & 30.8640 & 1.341 & 32.6929 & 1.215 & 32.8188 & 1.081 & 32.9364 \\
\hline 1.435 & 30.7416 & 1.055 & 30.8698 & 1.401 & 32.7002 & 1.195 & 32.8247 & 1.087 & 32.9422 \\
\hline 1.418 & 30.7481 & 1.055 & 30.8757 & 1.460 & 32.7062 & 1.168 & 32.8311 & 1.096 & 32.9482 \\
\hline 1.372 & 30.7569 & 1.063 & 30.8824 & 1.570 & 32.7171 & 1.159 & 32.8375 & 1.116 & 32.9540 \\
\hline 1.326 & 30.7635 & 1.066 & 30.8882 & 1.654 & 32.7254 & 1.133 & 32.8439 & 1.163 & 32.9698 \\
\hline 1.276 & 30.7704 & 1.079 & 30.8941 & 1.730 & 32.7321 & 1.110 & 32.8499 & 1.182 & 32.9757 \\
\hline 1.245 & 30.7769 & 1.097 & 30.9045 & 1.749 & 32.7379 & 1.089 & 32.8557 & & \\
\hline 1.203 & 30.7836 & 1.108 & 30.9104 & 1.744 & 32.7446 & 1.079 & 32.8616 & & \\
\hline$\Delta R$ & $\begin{array}{c}R_{\mathrm{HJD}} \\
2455800+\end{array}$ & $\Delta R$ & $\begin{array}{c}R_{\mathrm{HJD}} \\
2455800+\end{array}$ & $\Delta R$ & $\begin{array}{c}R_{\mathrm{HJD}} \\
2455800+\end{array}$ & $\Delta R$ & $\begin{array}{c}R_{\mathrm{HJD}} \\
2455800+\end{array}$ & $\Delta R$ & $\begin{array}{c}R_{\mathrm{HJD}} \\
2455800+\end{array}$ \\
\hline 1.163 & 30.6536 & 1.222 & 30.7908 & 1.188 & 30.9285 & 1.735 & 32.7509 & 1.108 & 32.8620 \\
\hline 1.198 & 30.6608 & 1.177 & 30.7977 & 1.205 & 30.9344 & 1.741 & 32.7579 & 1.098 & 32.8678 \\
\hline 1.227 & 30.6681 & 1.170 & 30.8042 & 1.238 & 30.9415 & 1.720 & 32.7638 & 1.090 & 32.8761 \\
\hline 1.261 & 30.6754 & 1.150 & 30.8109 & 1.145 & 32.6468 & 1.657 & 32.7702 & 1.081 & 32.8819 \\
\hline 1.312 & 30.6835 & 1.133 & 30.8175 & 1.172 & 32.6550 & 1.595 & 32.7760 & 1.073 & 32.8890 \\
\hline 1.355 & 30.6913 & 1.143 & 30.8256 & 1.191 & 32.6623 & 1.511 & 32.7825 & 1.069 & 32.8948 \\
\hline 1.419 & 30.6997 & 1.104 & 30.8364 & 1.213 & 32.6682 & 1.458 & 32.7883 & 1.078 & 32.9008 \\
\hline 1.463 & 30.7074 & 1.097 & 30.8429 & 1.241 & 32.6749 & 1.394 & 32.7957 & 1.074 & 32.9066 \\
\hline 1.481 & 30.7148 & 1.086 & 30.8508 & 1.291 & 32.6809 & 1.339 & 32.8014 & 1.085 & 32.9126 \\
\hline 1.480 & 30.7213 & 1.089 & 30.8566 & 1.330 & 32.6875 & 1.300 & 32.8076 & 1.085 & 32.9184 \\
\hline 1.486 & 30.7283 & 1.085 & 30.8644 & 1.372 & 32.6935 & 1.266 & 32.8134 & 1.098 & 32.9244 \\
\hline 1.494 & 30.7349 & 1.084 & 30.8762 & 1.422 & 32.7007 & 1.252 & 32.8193 & 1.101 & 32.9302 \\
\hline 1.482 & 30.7422 & 1.092 & 30.8828 & 1.491 & 32.7067 & 1.216 & 32.8251 & 1.106 & 32.9368 \\
\hline 1.464 & 30.7488 & 1.095 & 30.8887 & 1.584 & 32.7175 & 1.205 & 32.8316 & 1.119 & 32.9426 \\
\hline 1.410 & 30.7576 & 1.103 & 30.8946 & 1.680 & 32.7258 & 1.190 & 32.8380 & 1.152 & 32.9545 \\
\hline 1.357 & 30.7641 & 1.129 & 30.9050 & 1.738 & 32.7325 & 1.155 & 32.8444 & 1.189 & 32.9703 \\
\hline 1.313 & 30.7710 & 1.146 & 30.9109 & 1.741 & 32.7383 & 1.135 & 32.8504 & 1.215 & 32.9761 \\
\hline 1.286 & 30.7776 & 1.151 & 30.9167 & 1.734 & 32.7451 & 1.120 & 32.8562 & 1.179 & 32.9763 \\
\hline 1.242 & 30.7842 & 1.171 & 30.9227 & & & & & & \\
\hline$\Delta I$ & $\begin{array}{c}I_{\mathrm{HJD}} \\
2455800+ \\
\end{array}$ & $\Delta I$ & $\begin{array}{c}I_{\mathrm{HJD}} \\
2455800+ \\
\end{array}$ & $\Delta I$ & $\begin{array}{c}I_{\mathrm{HJD}} \\
2455800+ \\
\end{array}$ & $\Delta I$ & $\begin{array}{c}I_{\mathrm{HJD}} \\
2455800+ \\
\end{array}$ & $\Delta I$ & $\begin{array}{c}I_{\mathrm{HJD}} \\
2455800+ \\
\end{array}$ \\
\hline 1.181 & 30.6541 & 1.236 & 30.7913 & 1.224 & 30.9289 & 1.745 & 32.7583 & 1.135 & 32.8765 \\
\hline 1.210 & 30.6613 & 1.212 & 30.7982 & 1.247 & 30.9348 & 1.736 & 32.7642 & 1.124 & 32.8823 \\
\hline 1.238 & 30.6687 & 1.191 & 30.8048 & 1.264 & 30.9419 & 1.669 & 32.7706 & 1.118 & 32.8952 \\
\hline 1.274 & 30.6759 & 1.177 & 30.8114 & 1.161 & 32.6473 & 1.608 & 32.7764 & 1.108 & 32.9011 \\
\hline 1.315 & 30.6841 & 1.161 & 30.8180 & 1.211 & 32.6553 & 1.538 & 32.7829 & 1.113 & 32.9070 \\
\hline 1.372 & 30.6918 & 1.141 & 30.8261 & 1.227 & 32.6626 & 1.479 & 32.7887 & 1.118 & 32.9130 \\
\hline 1.426 & 30.7002 & 1.138 & 30.8369 & 1.239 & 32.6686 & 1.416 & 32.7961 & 1.131 & 32.9188 \\
\hline 1.476 & 30.7079 & 1.129 & 30.8434 & 1.257 & 32.6752 & 1.368 & 32.8019 & 1.148 & 32.9248 \\
\hline 1.486 & 30.7153 & 1.120 & 30.8512 & 1.329 & 32.6812 & 1.332 & 32.8080 & 1.145 & 32.9306 \\
\hline 1.501 & 30.7218 & 1.113 & 30.8570 & 1.348 & 32.6878 & 1.296 & 32.8138 & 1.148 & 32.9372 \\
\hline 1.501 & 30.7288 & 1.104 & 30.8648 & 1.402 & 32.6938 & 1.274 & 32.8197 & 1.155 & 32.9430 \\
\hline 1.506 & 30.7354 & 1.121 & 30.8707 & 1.452 & 32.7011 & 1.250 & 32.8255 & 1.168 & 32.9491 \\
\hline 1.516 & 30.7427 & 1.129 & 30.8832 & 1.510 & 32.7071 & 1.232 & 32.8320 & 1.184 & 32.9549 \\
\hline 1.485 & 30.7493 & 1.138 & 30.8891 & 1.606 & 32.7179 & 1.227 & 32.8384 & 1.240 & 32.9707 \\
\hline 1.424 & 30.7581 & 1.136 & 30.8950 & 1.689 & 32.7262 & 1.196 & 32.8448 & 1.253 & 32.9765 \\
\hline 1.379 & 30.7646 & 1.157 & 30.9054 & 1.733 & 32.7329 & 1.177 & 32.8508 & & \\
\hline 1.345 & 30.7715 & 1.174 & 30.9113 & 1.766 & 32.7387 & 1.164 & 32.8566 & & \\
\hline 1.286 & 30.7781 & 1.185 & 30.9172 & 1.748 & 32.7455 & 1.157 & 32.8624 & & \\
\hline 1.260 & 30.7847 & 1.206 & 30.9231 & 1.751 & 32.7513 & 1.134 & 32.8682 & & \\
\hline
\end{tabular}

HJD is the Heliocentric Julian Date. 


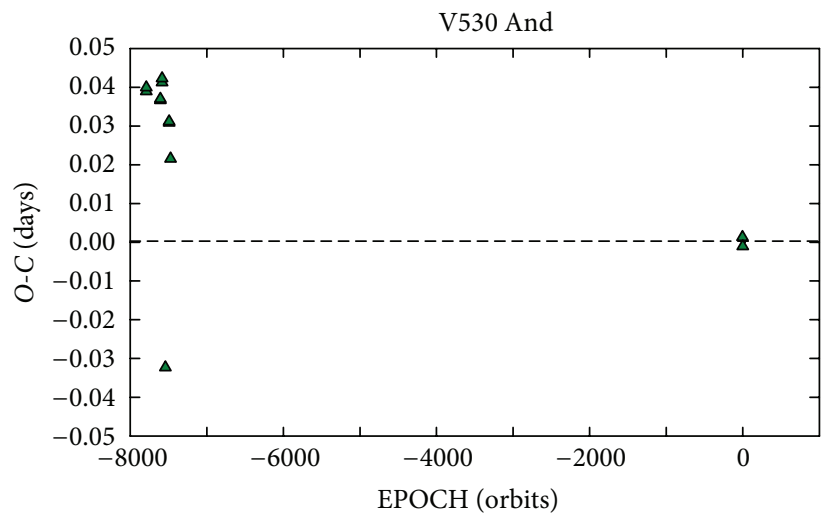

Figure 3: The $O-C$ residuals are calculated from (2) and appear in Table 2. The residuals on the left are low precision points with low weights from times of low light.

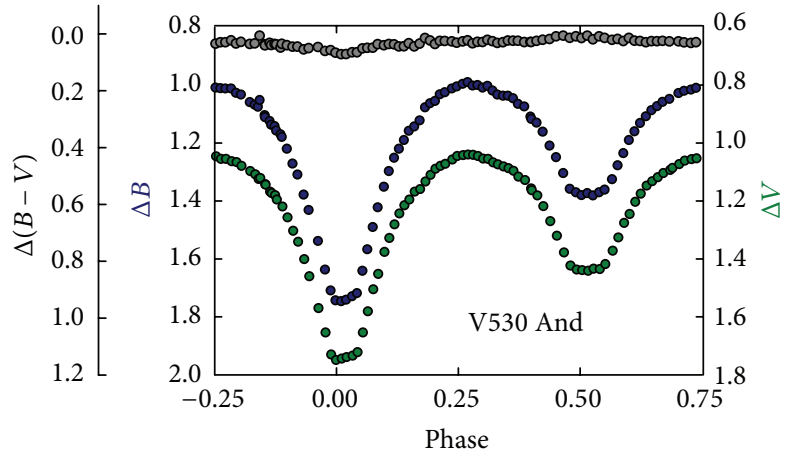

(a)

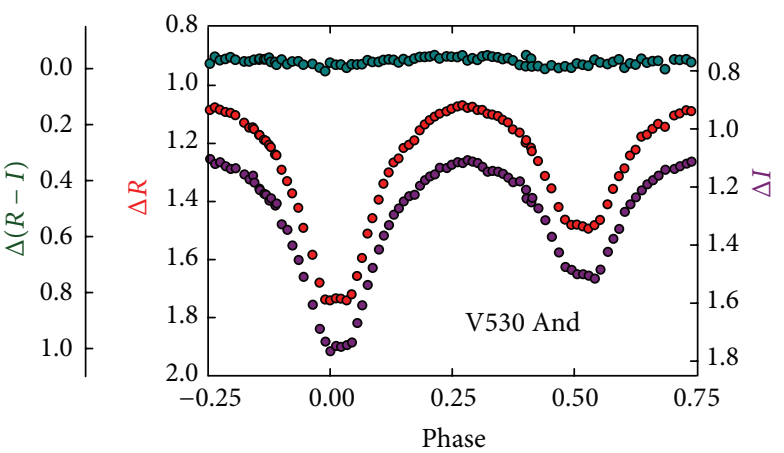

(b)

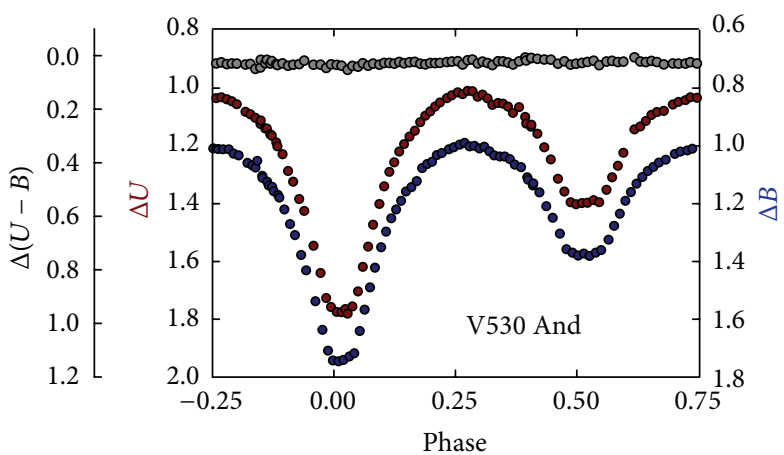

(c)

Figure 4: (a) $B, V$ delta mags of V530 And phased with (2). (b) $R, I$ delta mags of V530 and phased with (2). (c) $U, B$ delta mags of V530 And Phased with (2).

\section{Light Curves}

The light curves were folded $\Delta U, \Delta B, \Delta V, \Delta R, \Delta I, \Delta(U-B)$, $\Delta(B-V)$, and $\Delta(R-I)$ color curves using (2). They are given in Figures 4(a), 4(b), and 4(c).

Light curve characteristics are given in Table 3. The primary light curve amplitudes of this EB system average 0.8-0.6 mags from $U$ to $I$, respectively, and 0.4 mags in the secondary amplitudes. A small O'Connell effect (MAX IIMAX I) [3] of about $1 \%$ exists with MAX II being slightly depressed. Thus, we expect some spot activity. The secondary eclipse showed a time of constant light of 41.5 minutes. This means that the eclipses are total and that the more massive, larger star is the hotter component. This is to be expected in normal stellar evolution. We also note that this binary is not one of the common W-type shallow contact types.

\section{Synthetic Light Curve Solution}

2MASS Photometry yielded a $J-H$ value of $\approx 0.19$. From this, we assumed that the primary component was $\sim \mathrm{F} 2$ type, 


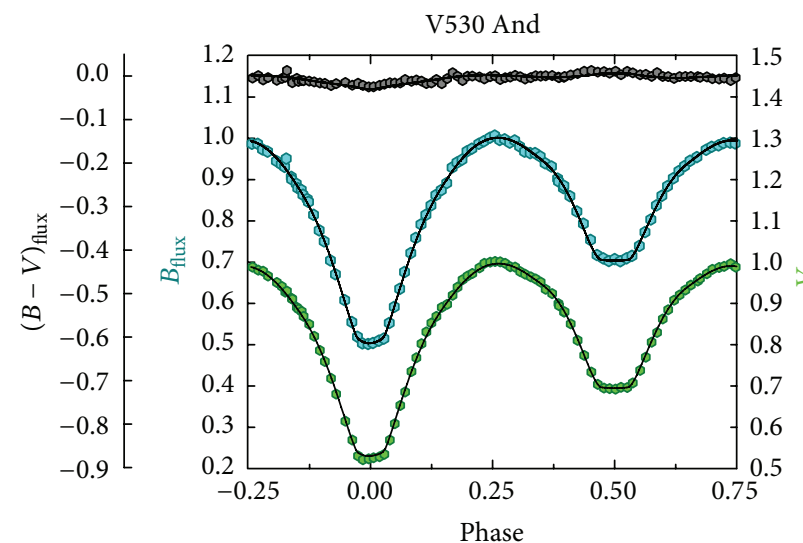

(a)

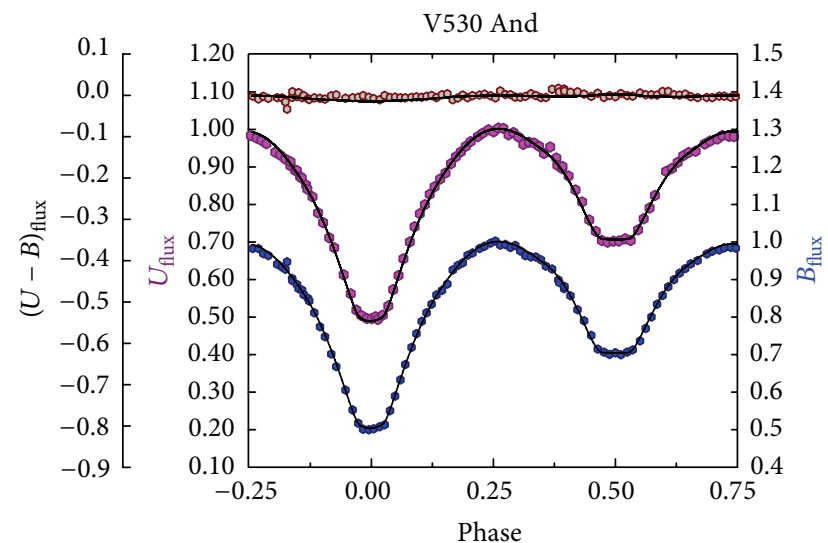

(b)

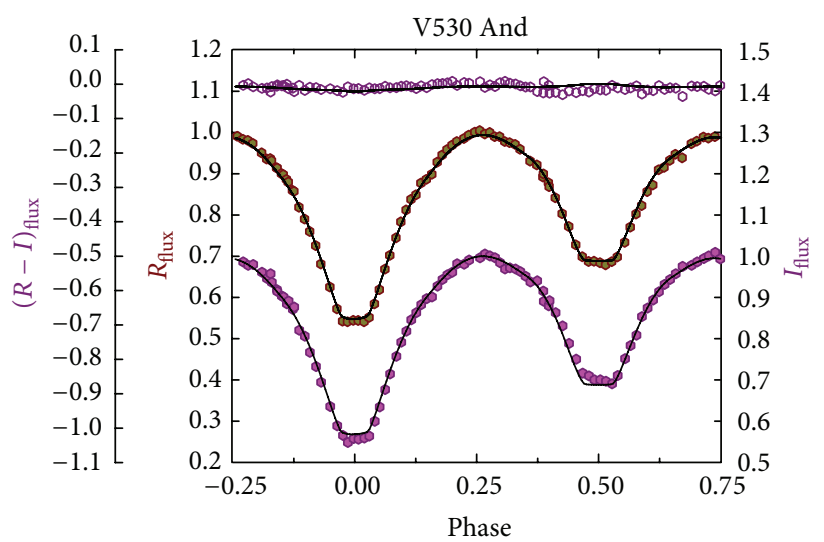

(c)

Figure 5: (a) $B, V$ synthetic light curve solutions overlaying the normalized flux curves. (b) $U, B$ synthetic light curve solutions overlaying the normalized flux curves. (c) $R, I$ synthetic light curve solutions overlaying the normalized flux curves.

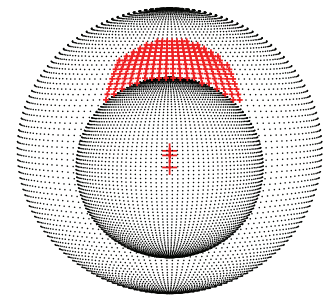

(a)

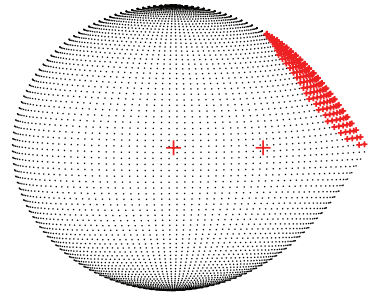

(b)

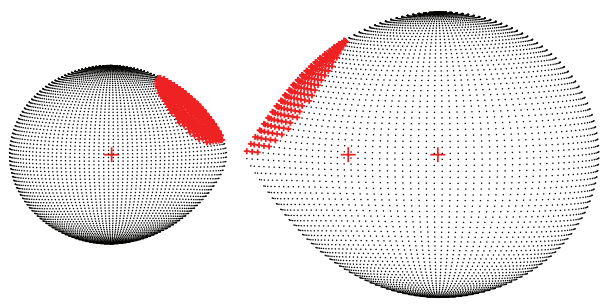

(d)
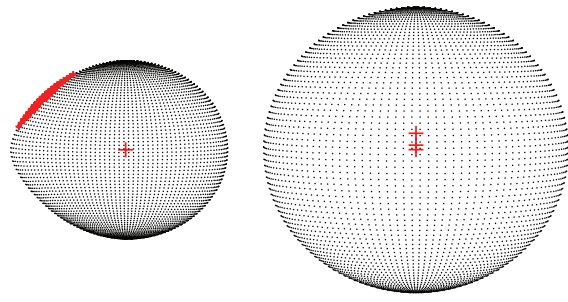

(c)

FIGURE 6: (a) Geometrical surface of V530 And, phase 0.0. (b) Geometrical surface of V530 And, phase 0.25. (c) Geometrical surface of V530 And, phase 0.50. Note total eclipse. (d) Geometrical surface of V530 And, phase 0.75. 
TABLE 2: Times of minimum light, V530 And.

\begin{tabular}{lccccc}
\hline No. & EPOCHS & Cycles & Weight & O-C & \\
\hline 1 & 51335.3990 & -7791.5 & 0.1 & 0.0390 & NSVS \\
2 & 51335.4000 & -7791.5 & 0.1 & 0.0400 & NSVS \\
3 & 51442.1819 & -7606.5 & 0.1 & 0.0367 & NSVS \\
4 & 51442.1822 & -7606.5 & 0.1 & 0.0370 & NSVS \\
5 & 51454.3080 & -7585.5 & 0.1 & 0.0413 & NSVS \\
6 & 51454.3091 & -7585.5 & 0.1 & 0.0423 & NSVS \\
7 & 51507.4016 & -7493.5 & 0.1 & 0.0309 & NSVS \\
8 & 51507.4019 & -7493.5 & 0.1 & 0.0312 & NSVS \\
9 & 51518.3594 & -7474.5 & 0.1 & 0.0216 & NSVS \\
10 & 51479.6320 & -7541.5 & 1.0 & -0.0323 & Khruslov (2008) [1] \\
11 & 55830.7281 & -3.5 & 1.0 & 0.0013 & Present observations \\
12 & 55832.7460 & 0.0 & 1.0 & -0.0010 & Present observations \\
\hline
\end{tabular}

TABLE 3: Light curve characteristics, V530 And.

\begin{tabular}{|c|c|c|c|c|}
\hline Filter & Phase 0.25 & MAX I, MAG & Phase 0.75 & MAX II, MAG \\
\hline$U$ & & $1.017 \pm 0.008$ & & $1.033 \pm 0.002$ \\
\hline$B$ & & $0.999 \pm 0.006$ & & $1.001 \pm 0.001$ \\
\hline$V$ & & $1.043 \pm 0.002$ & & $1.053 \pm 0.004$ \\
\hline$R$ & & $1.075 \pm 0.004$ & & $1.086 \pm 0.002$ \\
\hline$I$ & & $1.114 \pm 0.004$ & & $1.115 \pm 0.007$ \\
\hline Filter & Phase 0.5 & MIN II, MAG & Phase 0.0 & MIN I, MAG \\
\hline $\bar{U}$ & & $1.396 \pm 0.006$ & & $1.774 \pm 0.007$ \\
\hline$B$ & & $1.375 \pm 0.005$ & & $1.729 \pm 0.009$ \\
\hline$V$ & & $1.437 \pm 0.003$ & & $1.740 \pm 0.006$ \\
\hline$R$ & & $1.485 \pm 0.005$ & & $1.738 \pm 0.003$ \\
\hline$I$ & & $1.502 \pm 0.010$ & & $1.754 \pm 0.008$ \\
\hline Filter & MIN I-MAX I & MAX II-MAX I & MIN II-MAX I & MIN I-MIN II \\
\hline$U$ & $0.757 \pm 0.015$ & $0.016 \pm 0.010$ & $0.379 \pm 0.014$ & $0.378 \pm 0.013$ \\
\hline$B$ & $0.730 \pm 0.015$ & $0.003 \pm 0.007$ & $0.376 \pm 0.011$ & $0.354 \pm 0.014$ \\
\hline$V$ & $0.697 \pm 0.008$ & $0.010 \pm 0.006$ & $0.394 \pm 0.005$ & $0.303 \pm 0.009$ \\
\hline$R$ & $0.663 \pm 0.008$ & $0.011 \pm 0.006$ & $0.410 \pm 0.009$ & $0.253 \pm 0.008$ \\
\hline$I$ & $0.640 \pm 0.013$ & $0.001 \pm 0.012$ & $0.388 \pm 0.014$ & $0.252 \pm 0.018$ \\
\hline
\end{tabular}

with a surface temperature of $\approx 7000 \mathrm{~K}$ [4] so this value was used for the primary components temperature in modeling. The light curves were premodeled with Binary Maker 3.0 [5]. From this work, it was determined that the synthetic light curve fits would be done in Mode 4, semidetached with component one filling its Roche lobe. The parameters resulting from this premodeling were used as starting values for the Wilson-Devinney program [6-9]. The secondary component remained under-filling its Roche lobe throughout the iterations. Adjusted parameters were those with parentheses in Table 4. A simultaneous 5-color synthetic light curve solution was undertaken with the Wilson-Devinney program. No q-search was needed since the curves display total eclipses. A mode 3 solution (contact binary) was also run with much poorer goodness of fit parameters.

\section{Discussion}

We find that V530 And is a V1010 Oph type near contact binary. The primary, more massive component is filling its Roche lobe and the secondary is under-filling. This means that the binary is likely achieving contact for the first time. The temperature difference $\left(T_{2}-T_{1}\right)$ in components is $\sim 500 \mathrm{~K}$. The mass ratio was found to be somewhat extreme, $q=m_{1} / m_{2}=0.38$. The potentials are given as $\Omega / \mathrm{s}$, and the $L$-values are the relative flux from each stars. The values of $g$ are the gravity darkening's, $x$ 's are the two dimensional limb darkening, and the $r^{\prime}$ s are the dimensions of the Roche lobes. The more massive star is filling its Roche lobe with a fillout, $f$, of $100 \%$ while the smaller star is just under-filling with a fillout of $99 \%$ calculated by potentials. Thus, the state 
TABLE 4: Light curve solution, V530 And.

\begin{tabular}{|c|c|}
\hline Parameters & Values \\
\hline$\lambda_{U}, \lambda_{B}, \lambda_{V}, \lambda_{R}, \lambda_{I}(\mathrm{~nm})$ & $360,440,550,640,790$ \\
\hline$x_{\text {boll }, 2}, y_{\text {boll,2 }}$ & $0.644,0.643,0.231,0.160$ \\
\hline$x_{1 I, 2 I}, y_{1 I, 2 I}$ & $0.572,0.647,0.267,0.183$ \\
\hline$x_{1 R, 2 R}, y_{1 R, 2 R}$ & $0.655,0.735,0.278,0.165$ \\
\hline$x_{1 V, 2 V}, y_{1 V, 2 V}$ & $0.728,0.797,0.269,0.108$ \\
\hline$x_{1 B, 2 B}, y_{1 B, 2 B}$ & $0.817,0.853,0.215,-0.018$ \\
\hline$x_{1 U, 2 U}, y_{1 U, 2 U}$ & $0.859,0.855,0.208,-0.209$ \\
\hline$g_{1}, g_{2}$ & 0.32 \\
\hline$A_{1}, A_{2}$ & 0.5 \\
\hline Inclination $\left(^{\circ}\right)$ & $87.1 \pm 0.2$ \\
\hline$T_{1}, T_{2}(\mathrm{~K})$ & $7000,6499 \pm 2$ \\
\hline$\Omega_{1}, \Omega_{2}$ & $2.63423,2.662 \pm 0.001$ \\
\hline$q\left(m_{2} / m_{1}\right)$ & $0.3786 \pm 0.0003$ \\
\hline Fill-outs: $F_{1}=F_{2}$ & $100 \%, 98.97 \pm 0.72 \%$ \\
\hline$L_{1} /\left(L_{1}+L_{2}\right)_{I}$ & $0.7687 \pm 0.0007$ \\
\hline$L_{1} /\left(L_{1}+L_{2}\right)_{R}$ & $0.7790 \pm 0.0008$ \\
\hline$L_{1} /\left(L_{1}+L_{2}\right)_{V}$ & $0.790 \pm 0.001$ \\
\hline$L_{1} /\left(L_{1}+L_{2}\right)_{B}$ & $0.8064 \pm 0.0008$ \\
\hline$L_{1} /\left(L_{1}+L_{2}\right)_{U}$ & $0.8140 \pm 0.0006$ \\
\hline $\mathrm{JD}_{\mathrm{o}}$ (days) & $55832.7468 \pm 0.0001$ \\
\hline Period (days) & $0.57680 \pm 0.00006$ \\
\hline$r_{1}, r_{2}$ (pole) & $0.437 \pm 0.001,0.273 \pm 0.005$ \\
\hline$r_{1}, r_{2}$ (point) & $0.598 \pm 0.001,0.35 \pm 0.02$ \\
\hline$r_{1}, r_{2}($ side $)$ & $0.467 \pm 0.002,0.284 \pm 0.006$ \\
\hline \multirow[t]{2}{*}{$r_{1}, r_{2}$ (back) } & $0.494 \pm 0.001,0.313 \pm 0.009$ \\
\hline & Cool Spot \\
\hline \multicolumn{2}{|l|}{ Spot 1 on STAR 1} \\
\hline Colatitude $\left({ }^{\circ}\right)$ & $64.1 \pm 0.3$ \\
\hline Longitude $\left(^{\circ}\right)$ & $1.1 \pm 0.2$ \\
\hline Spot radius $\left({ }^{\circ}\right)$ & $26.0 \pm 0.1$ \\
\hline Spot $T$-factor & $0.694 \pm 0.004$ \\
\hline \multicolumn{2}{|l|}{ Spot 2 on STAR 1} \\
\hline Colatitude $\left(^{\circ}\right)$ & $58 \pm 7$ \\
\hline Longitude $\left({ }^{\circ}\right)$ & $346 \pm 2$ \\
\hline Spot radius $\left({ }^{\circ}\right)$ & $26 \pm 1$ \\
\hline Spot $T$-factor & $0.92 \pm 0.01$ \\
\hline
\end{tabular}

of the star is near contact or has just come into contact. We reason this is true because of the temperature disparity. It is of interest that both surface spots stayed remarkably stable throughout the iterations and are near the $L_{1}$ points of both stars. There may be some thermodynamic reason why the hemispheres of dark spots are facing each other. The solution is seen overlaying the normalized flux curves shown in Figures 5(a), 5(b), and 5(c). The complete numeric values for the solution are given in Table 4 . Four phases, at orbital quadrature's, of the Roche-lobe model of the binary and for the dark spots, are shown as in Figures 6(a), 6(b), 6(c) and 6(d). Phase zero shows the total eclipse.

\section{Conclusion}

Thus, the components are apparently F2V and F5V spectral types. Despite the fairly early spectral types, with the primary star nearly A-type, the presence of the large cool magnetic spots on both stars reveal that they have convective atmospheres and thus would be classified as solar type. It is a near contact, totally eclipsing, binary. Thus, the binary will likely soon come into contact through the process of magnetic braking due to solar type winds leaving the stars along the stiff magnetic field lines [10].

\section{Acknowledgments}

The authors thank USC, Lancaster, for their support of thier membership in NURO for the past 8 years, the American Astronomical Society for its support through its former small research program, and the Arizona Space Grant for the partial support for thier student's travel.

\section{References}

[1] A. V. Khruslov, "New short periodic eclipsing binaries. III," Peremennye Zvezdy Prilozhenie, vol. 8, no. 40, 2008.

[2] D. I. Hoffman, T. E. Harrison, and B. J. McNamara, "Automated variable star classification using the northern sky variability survey," Astronomical Journal, vol. 138, no. 2, pp. 466-477, 2009.

[3] D. J. K. O'Connell, “The so-called periastron effect in close eclipsing binaries," Riverview College Observatory Publications, vol. 2, no. 6, pp. 85-100, 1951.

[4] A. N. Cox, Ed., Allen's Astrophysical Quantities, Springer, New York, NY, USA, 4th edition, 2000.

[5] D. H. Bradstreet and D. P. Steelman, "Binary maker 3.0an interactive graphics-based light curve synthesis program written in Java," Bulletin of the American Astronomical Society, vol. 34, p. 1224, 2002.

[6] R. E. Wilson and E. J. Devinney, "Realization of accurate closebinary light curves: application to MR Cygni," Astrophysical Journal, vol. 166, pp. 605-619, 1971.

[7] R. E. Wilson, "Accuracy and efficiency in the binary star reflection effect," Astrophysical Journal Letters, vol. 356, no. 2, pp. 613622, 1990.

[8] R. E. Wilson, "Binary-star light curve models," Publications of the Astronomical Society of the Pacific, vol. 106, pp. 921-941, 1994.

[9] W. V. van Hamme and R. E. Wilson, "Generalized solution for binary star ephemerides and apsidal motion," Bulletin of the American Astronomical Society, vol. 30, p. 1402, 1998.

[10] E. F. Guinan and D. H. Bradstreet, "Kinematic clues to the origin and evolution of low mass contact binaries, formation and evolution of low mass stars," in Proceedings of the NATO Advanced Study Institute, Held at Viana do Castelo, Portugal, September 21-October 2, 1987, A. K. Dupree and M. T. V. T. Lago, Eds., vol. 241 of NATO Advanced Science Institutes (ASI) Series C, pp. 345-375, Kluwer Academic, Dodrecht, The Netherlands, 1988. 

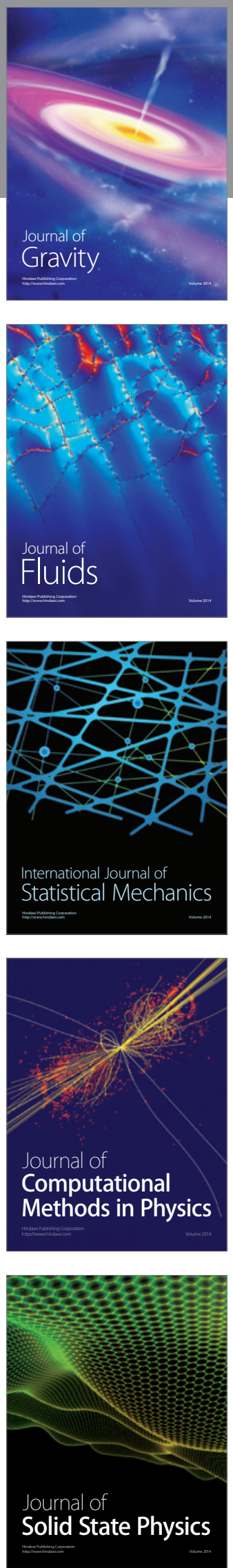

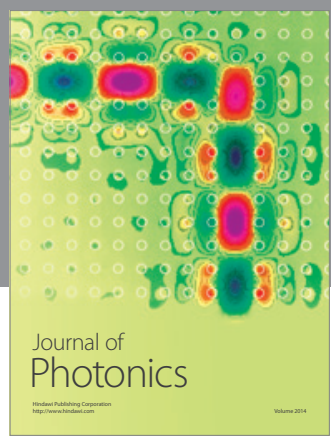

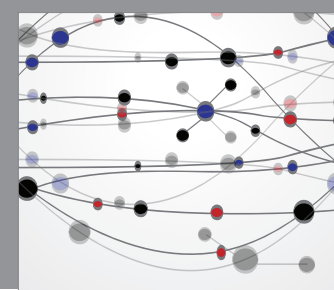

The Scientific World Journal

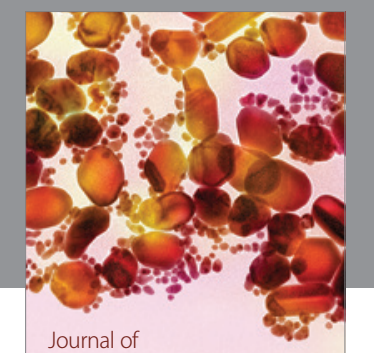

Soft Matter
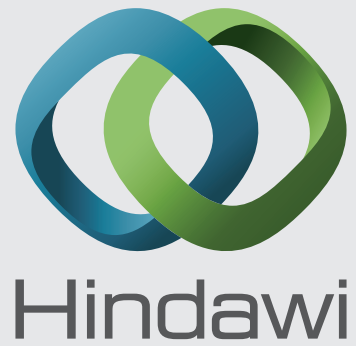

Submit your manuscripts at

http://www.hindawi.com
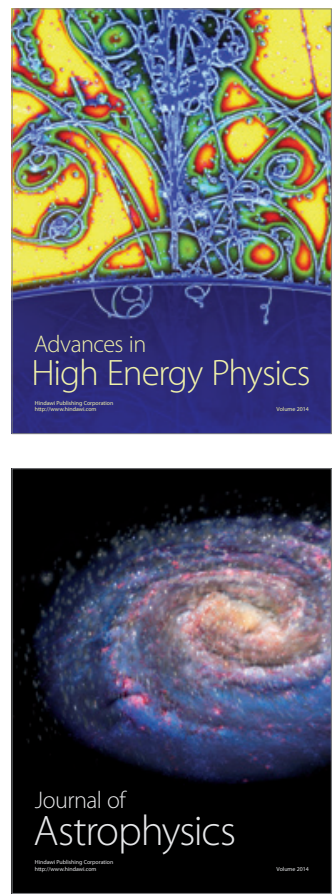
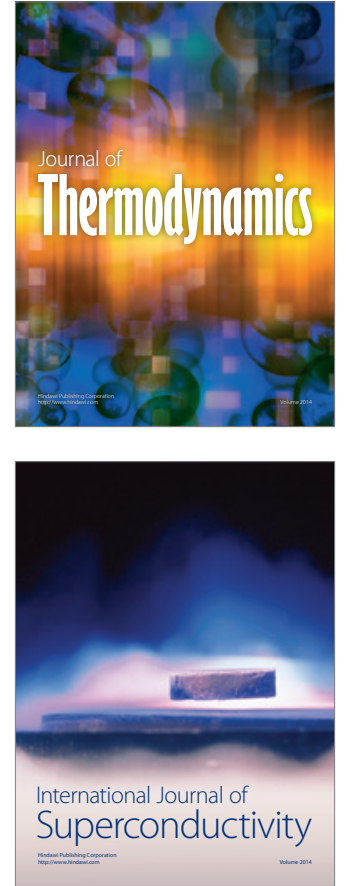
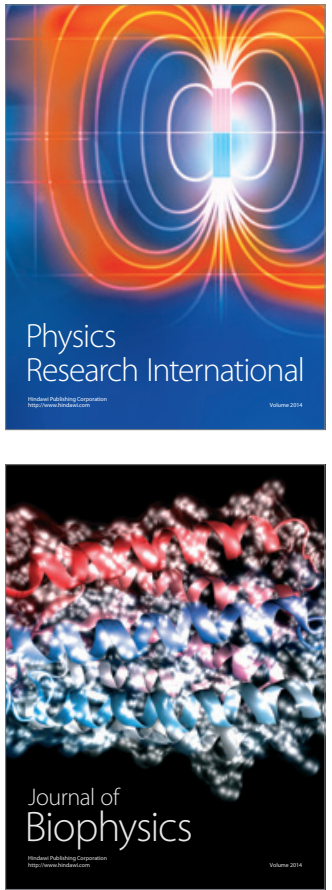
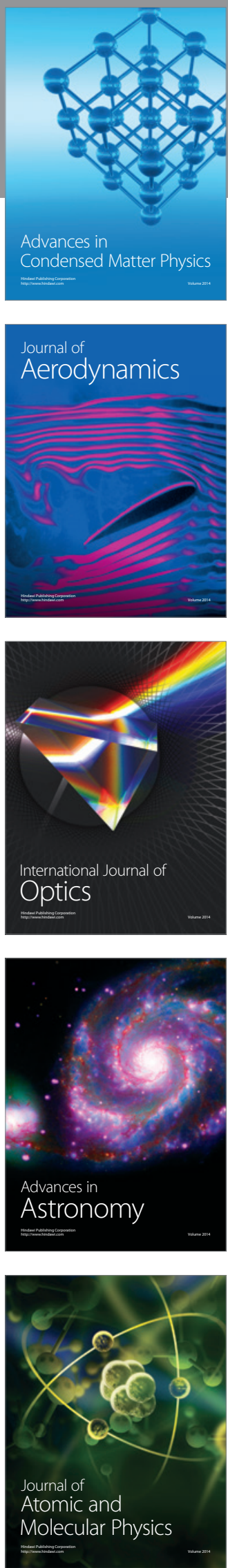